\title{
An Unusual Supraclavicular Mass in a Treated Early Breast Cancer Patient: A Case Report David Okonji ${ }^{1 *}$, Michael McCrystal ${ }^{2}$ and Jan Craik ${ }^{3}$
}

\author{
*Corresponding author: David Okonji
}

Address: ${ }^{1}$ Department of Medical Oncology, Wellington Regional Hospital, Wellington, New Zealand; ${ }^{2}$ Department of Medical Oncology, New Zealand; ${ }^{3}$ Auckland City Hospital, Auckland; Department of Histopathology and Cytology, Northshore Hospital, Auckland, New Zealand e-mail $₫$ david.okonji@ccdhb.org.nz

Received: 17 July 2020; Accepted: 24 July 2020

\section{ABSTRACT}

Isolated contralateral supraclavicular lymphadenopathy following adjuvant breast cancer treatment is an uncommon presentation and should be regarded as highly suspicious for the development of Stage IV disease. Fine needle aspiration (FNA) is a simple and inexpensive method of confirming the diagnosis. We report a case of a 42 year old lady with treated early breast cancer presenting with an isolated supraclavicular mass. The key issues supporting a cautious approach prior FNA are discussed.

Keywords: Supraclavicular lymphadenopathy, Diagnosis, Investigation

\section{Introduction}

Breast Cancer commonly metastasizes to the ipsilateral supraclavicular lymph node basin, with contralateral involvement considered to be Stage IV disease with all the attendant consequences of this diagnosis.

With a history of resected, intermediate risk early breast cancer treated with adjuvant chemotherapy, radiotherapy and endocrine therapy, the clinical evidence of presentation with delayed contralateral supraclavicular lymphadenopathy must be considered with a high suspicion for recurrence and is an unfavourable prognostic indicator (Debois et al., 1997). However, obtaining a complete history and requesting appropriate investigations, including pathological evaluation of a tissue biopsy is important in determining the definitive diagnosis.

We report a case of what was initially thought to be breast cancer recurrence with isolated contralateral supraclavicular lymphadenopathy.

\section{Clinical Practice Points}

- The development of isolated contralateral supraclavicular lymphadenopathy in the setting of early breast cancer, although uncommon, is highly suggestive of metastatic disease. 
- A careful history, examination and appropriate investigations should be undertaken first prior to invasive diagnostic procedures.

\section{Case Report}

A 42 year-old lady who was brought up in a sheep and cattle farm in Wairarapa in New Zealand moved to Auckland City in 1995. Four years later in 1999, she presented with a right upper quadrant right breast mass. U/S guided biopsy of the mass on 18/04/1999 confirmed early breast cancer.

On 06/05/1999, she had a right partial mastectomy and axillary lymph node dissection. Histology showed a Grade 2, ER+/PR+, 27 mm moderately differentiated infiltrating ductal carcinoma with $\mathrm{o} / 17$ lymph nodes involved. There was no vascular invasion nor extensive intraductal component. Ductal carcinoma in situ was present at $3 \mathrm{~mm}$ from deep margin. Staging CT showed no distant metastases in liver, lung or spleen. She completed adjuvant chemotherapy with six cycles of cyclophosphamide, methotrexate and 5-Fluoruracil followed by adjuvant radiotherapy to the right chest wall and axilla on 07/12/1999. She commenced adjuvant tamoxifen in Feb 2000.

A year later, in June 2001, in Medical Oncology Clinic follow-up clinic she was found to have "fullness in the left supraclavicular fossa. On the basis of a high clinical suspicion of malignant relapse, an ultrasound-guided fine needle aspiration (FNA) of the lesion was requested almost as a formality. The patient was warned of a high likelihood of metastatic disease. Microscopy following FNA biopsy on 15/06/2001 revealed a "background of flocculent amorphous debris together with brood capsules and scolices with hooklets consistent with echinococcal infection. No malignant cells were seen”.

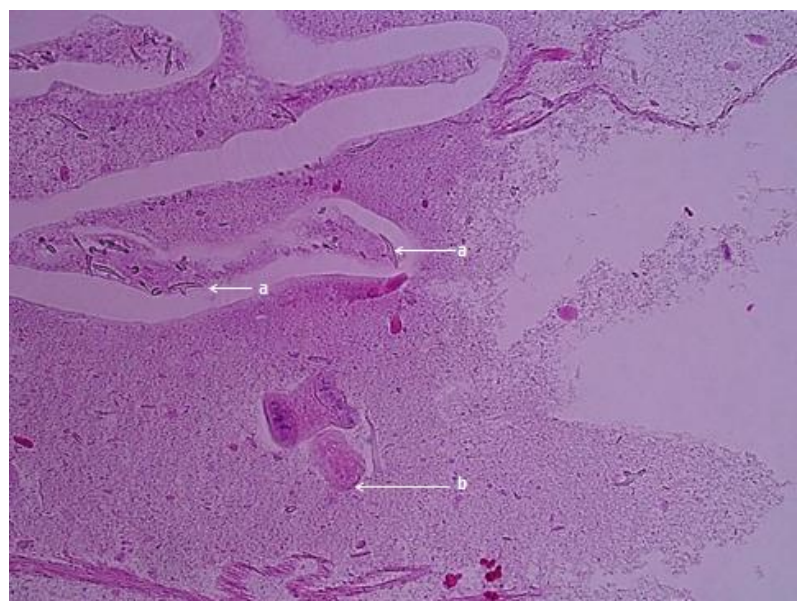

Figure 1: Hydatid scolex hooklets (b) and scolex (b)

Her FBC showed normal parameters except for moderate neutropenia at 1.85. She had a normal eosinophil count. Hydatid serology was negative. A subsequent Staging MRI Body 
showed "a hydatid cyst with multiple daughter cysts situated just above the brachial plexus in the supraclavicular fossa.

She was started on neoadjuvant antibiotic therapy with albendazole $900 \mathrm{mg} /$ day for one month. In 16/08/2001 she had surgical resection of the hydatid cyst.

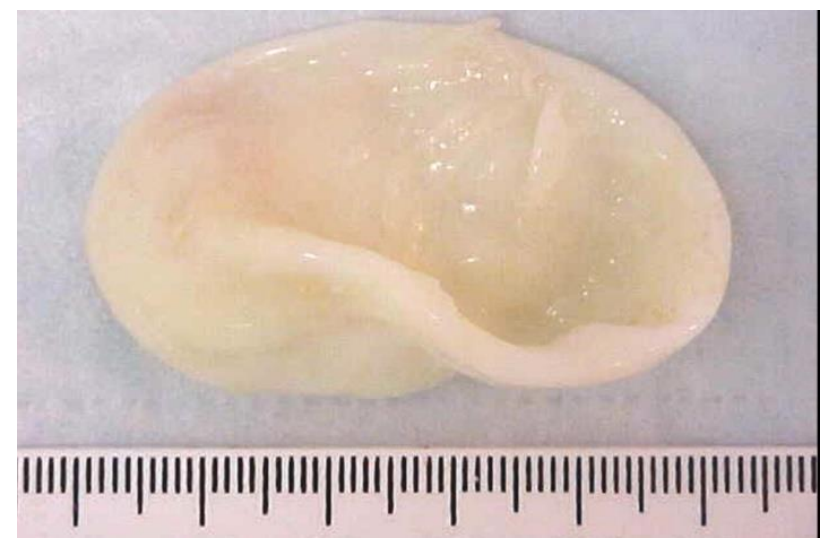

Figure 2: Excised hydatid cyst

At 13 months post-surgical excision of hydatid cyst, follow-up ultrasound scan of the left supraclavicular fossa on 29/11/o1 showed no residual or relapsed hydatid cysts.

\section{Discussion}

The prevalence of isolated contralateral supraclavicular lymphadenopathy as a presentation of recurrent breast cancer is uncommon and is estimated to be about $1-3 \%$ in one large case series (Van der Sangen et al., 2003). It is therefore important to ultimately confirm the diagnosis of recurrence with an excision or fine needle aspiration biopsy to establish the receptor status of the metastatic disease to guide future therapy (Foukakis et al., 2012). However, this report highlights the importance of careful history and selective radiological imaging prior to proceeding with fine needle aspiration biopsy of what may appear to be a 'lymph node': in our case this was an unusual finding of a solitary supraclavicular hydatid cyst.

Hydatid cysts are secondary to a zoonotic infection caused by Echinococcus species. It is endemic in rural cattle and sheep rearing communities in temperate climates in South America, parts of Africa, the Mediterranean, and Central Asia. However, in Tasmania and New Zealand, it has been declared 'provisionally eradicated" (Moro and Schantz, 2010).

In endemic areas, the incidence of primary solitary subcutaneous hydatid cysts without any visceral involvement is rare and has been estimated to be between $0.6-2.6 \%$ according to large case series (Kayaalp et al., 2011). Only 8.7\% of these involve the upper extremities (Vecchio et al., 2013). Furthermore, presentation of hydatid disease in the supraclavicular region is extremely rare (Nandy et al., 2012). Eosinophilia and parasitological serology are seldom positive (Foukakis et al., 2012). Diagnosis is primarily from the history and radiological 
imaging. Fine needle aspiration as a diagnostic tool is generally not recommended due to the risk of anaphylaxis or disseminated disease due to spillage of protoscolices. Fortunately, this did not occur in our patient and subsequent surgical resection and antibiotic therapy was deployed as is the recommended treatment.

The rarity of our case is further supported by the low prevalence of solid cancer seen in those with concomitant hydatid disease even in countries where the latter is endemic. Akgül and colleagues, in their large 12 year retrospective case series of patients undergoing surgical resection of solid tumours demonstrated a prevalence in coexistence of hydatid and cancer of 0.2\% (Akgül et al., 2003). This phenomenon may be due to the antigenic similarity has been reported between certain tumour types and Echinococcal cysts (Van Knapen, 1980). Cancerassociated O-glycosylated Th antigen is seen in the early phases of carcinogensis in various solid tumours including breast cancer (Springer, 1997). Tn antigen seen in larval and adult tissues of Echinococcus granulosus has been shown to be strikingly similar to cancerassociated O-glycosylated Tn antigen (Alvarez Errico et al., 2001). In fact one study, demonstrated a cross-reactive immune response resulting in antitumour activity when antigen-containing human hydatid cyst fluid was introduced into a murine model of colon cancer (Berriel et al., 2013). This may provide an explanation for the low prevalence of the coexistence of cancer in those with hydatid disease.

\section{Conclusion}

In summary, this had an unusual presentation of hydatid disease in a non-endemic country in a patient with a background of early breast cancer. Although current evidence is retrospective, the coexistence of hydatid disease and malignancy is extremely rare. This case highlights that although relapsed metastatic disease requires histological confirmation, the approach and choice of intervention to establish the diagnosis requires careful thought and consideration.

\section{Disclosure}

The authors have no potential conflicts or financial relationships of interest to declare.

\section{References}

Akgül H, Tez M, Unal AE, Keşkek M, Sayek I, Ozçelik. “Echinococcus against cancer: why not?” Cancer 2003; 98: 1999-2000.

Alvarez Errico D, Medeiros A, Míguez, Casaravilla C, Malgor R, Carmona C, Nieto A, Osinaga E: Oglycosylation in Echinococcus granulosus: identification and characterization of the carcinoma-associated Tn antigen. Exp Parasitol 2001; 98: 10-109.

Berriel E, Russo, S, Monin L, Festari MF, Berois N, Fernández G, Freire T, Osinaga E: Antitumour activity of human hydatid cyst in a murine model of colon cancer. The Scientific World Journal 2013; 18: 1-7

Debois JM. The significance of a supraclavicular node metastasis in patients with breast cancer. A literature review. Strahlenther Onkol 1997; 173: 1-12. 
Foukakis T, Astrom G, Lindstrom L, Hatschok T, Bergh J. When to order a biopsy to characterise a metastatic relapse in breast cancer. Ann Oncol 2012; 23: 349-353. 9: $117-721$.

Kayaalp C, Dirican A, Aydin C. Primary subcutaneous hydatid cysts: a review of 22 cases. Int J Surg 2011;

Moro P and Schantz PM. Echinococcosis: a review. Int J Infect Dis 2010; 14: e638-e648

Nandy M, Chakrabarti A, Mallik S. Hydatid disease presenting as multiple cystic swelling in the right supraclavicular region. Asian Pac Journal of Tropical Diseases 2012; 2: 490-491.

Springer GF. Immunoreactive T and TN epitopes in cancer diagnosis, prognosis and immunotherapy. $J$ Mol Med 1997; 75: 594-602.

Van der Sangen MJC, Coebergh JWW, Roumen RMH, Rutten HJT, Vreugdenhill G, Voogd AC. Detection, Treatment and Outcome of isolated Supraclavicular Recurrence in 42 patients with Invasive Breast Cancer. Cancer 2003; 98: 11-17.

Van Knapen F. Echinococcus granulosos infection in malignancy. Br Med $J$ 1980; 281: 195-196.

Vecchio R, Marchese S, Ferla F, Spataro L, Intagliata E. Solitary subcutaneous hydatid cyst: review of the Literature and report of a new case in the deltoid region. Parasitol Int 2013; 62: 487-493. 